\title{
Controlling spherical mobile robot in a two-parametric friction model
}

\author{
Georgy Terekhov ${ }^{1 *}$ and Vladimir Pavlovsky ${ }^{2}$ \\ ${ }^{1}$ Moscow State University, Moscow, Russia \\ ${ }^{2}$ Keldysh Institute of Applied Mathematics of RAS, Moscow, Russia
}

\begin{abstract}
The theoretical mechanical model of the spherical robot with the flywheels located in a sphere is considered. The described design is intended for the motion control of a sphere on various trajectories. In the paper, control algorithms of a sphere on basic trajectories are investigated; the movements from rotation on the point and the movements on a straight line piece before the basic movements on curvilinear trajectories are considered. The paper describes dynamical models of a sphere robot, different models of friction, as well as a two-parametrical model as the main model of friction. Technical possibility of realization of a sphere robot with flywheels is proved.
\end{abstract}

\section{Introduction}

At present there is a significant variety of robots, differing in design and purpose. Among them, it is interesting to consider robots of spherical shape that have such advantages over other implementations as the tightness of the robot and the absence of junctions and articulations that are most vulnerable to various kinds of adverse effects. This form of the device contributes to the practical use of the robot for research and reconnaissance purposes.

Such devices are actively being developed in different countries of the world - Sweden [1], the USA, Israel, Russia, etc. A large number of constructions use the principle of moving the center of the mass of system, such as various pendulum robots, or robots with moving internal masses. The devices moving due to deformation of the case are also of interest. This paper is devoted to another realization of the gyrostat principle, which implies the presence of a system of drives inside the sphere, which provides the creation of an internal kinetic moment. In particular, we consider an example of a robot, inside of which three flywheels are located on three mutually orthogonal axes. These flywheels produce a control moment.

An investigation of the motion and planning of the trajectories of such a device on an absolutely rough plane was performed in [2]. Then, in [3] the controllability of the system for the nonholonomic system was proved and the robot control algorithms were explicitly indicated. In [4] the results were generalized to the case of a plane with friction, in particular, Coulomb's dry friction, viscous friction are considered.

At the same time, recently, a number of papers appear, suggesting essentially altered models of friction, which supplement and generalize the model of
Coulomb's dry friction. The generalization is due to the fact that the point of Coulomb's interaction of the rolling body with the plane is replaced by an interaction along a certain region (contact spots). An example of this approach is the consideration of the contact spot in the form of a circle, this model was first proposed by Contensou [5] and further developed by V.F. Zhuravlev [6]. With this approach, there are plane deformations of the spherical shell of the ball near the contact with an absolutely rigid plane. However, there is also an approach, according to which the contact spot is not a flat region, but a spherical segment. Thus, it is assumed deformation not only areas but also the reference plane. Such a model was proposed and then developed by A.V. Karapetyan in $[7,8]$. In this paper we use a twoparameter model of friction [7].

\section{Theoretical mechanical model}

Consider on a fixed plane $S x y z$ a sphere of mass M and radius $R$, in which three flywheel disks with radius $r$ and mass $\mathrm{m}$ with centers at the points $A_{i}(i=1,2,3)$ are located on mutually orthogonal axes. The distance from the point of intersection of the flywheel axes to each of $A_{i}$ is equal to $a$. The center of mass of the whole system is assumed at point $O$. Next, we move to dimensionless linear, mass and inertial parameters by dividing them by $R, M+3 m$ and $m a^{2} / 3$, respectively. We introduce the movable coordinate system $O e_{1} e_{2} e_{3}$, with the axes directed parallel to the flywheel axes. Theorems on the change in momentum and angular momentum take the form:

$$
v=f
$$

\footnotetext{
${ }^{*}$ Corresponding author: arctangens90@gmail.com
} 


$$
\begin{aligned}
& \frac{d}{d t}(I \omega+C \alpha)=D \mu . \\
& \mu=\mu_{0}-b\left[e_{z} ; f\right] .
\end{aligned}
$$

Here the first equation is written in the absolute coordinate system, and the second - in the movable coordinate system. The matrix $I$ is the inertia tensor of the system in a movable coordinate system. It is assumed that this matrix has the form:

$$
I=\left(\begin{array}{lll}
v & 1 & 1 \\
1 & v & 1 \\
1 & 1 & v
\end{array}\right)
$$

where $v>1$. Matrix $C$-diagonal matrix, consisting of values of axial moments of inertia of flywheels. The vectors $\boldsymbol{f}$ and $\boldsymbol{\mu}^{\mathbf{0}}$ are vectors of external forces and torques. The vector $\boldsymbol{\mu}-$ is the resultant vector of the torques. $D$-matrix of the transition from a fixed coordinate system to a moving one. $\boldsymbol{\Omega}$-vector of angular velocity in a moving coordinate system, $\boldsymbol{\alpha}$-angular velocity of rotation of flywheels around its axis, $\boldsymbol{v}$ velocity of the center of the robot in the absolute coordinate system. Further, to close the system, it is necessary to specify the conditions of contact interaction between the spherical surface of the robot and the reference plane.

\subsection{Two-parameter model of friction}

Real experiments show that contact between the spherical surface of the robot and the reference plane occurs not at one point, but in some region called the contact spot. The most precise is an assumption $[2,3]$ that it is a spherical segment, at each point of which the Coulomb dry friction law is locally satisfied. Let the radius of the sphere specifying this segment be $R_{f}$, and the radius of the segment is $R_{s}$.

Thus, the contact spot depends on the two parameters $\varepsilon$ and $\delta$, defined as $R_{s} / R$ and $R / R_{f}$, respectively. Both of these parameters take values from 0 to 1 . Expressions for forces and torques of friction take the following form:

$$
\begin{gathered}
f_{j}=\frac{3 k N \int_{0}^{\beta_{0}} \sqrt{\cos \beta-\cos \beta_{0}} \sin \beta I_{u_{j}} d \beta}{4 \pi(M+3 m) R\left(1-\cos \beta_{0}\right)^{\frac{3}{2}}}, \\
\mu_{j}{ }^{0}=\frac{3 k N \int_{0}^{\beta_{0}} \sqrt{\cos \beta-\cos \beta_{0}} \sin \beta I_{w_{j}} d \beta}{4 \pi \delta(M+3 m) R\left(1-\cos \beta_{0}\right)^{\frac{3}{2}}}, \\
I_{u_{j}}=\int_{0}^{2 \pi} \frac{u_{j}}{\|u\|} d \alpha . \\
I_{w_{j}}=\int_{0}^{2 \pi} \frac{w_{j}}{\|u\|} d \alpha .
\end{gathered}
$$

Here $g$ is the acceleration of gravity, $N$ is the force of the normal support reaction, $u$ is the slipping velocity of the spherical robot, divided by its radius, the first axis is directed along the slip velocity, the third axis is vertical, and the second axis completes the basis to the right triple. This basis will be referred to as auxiliary in the future, and vector will be indexed with Roman numerals to avoid confusion with coordinate system, rigidly connected with the robot. $\boldsymbol{\Omega}$ is the angular velocity vector in this basis. Finally, $\sin \beta_{0}=\frac{R_{s}}{R_{f}}=\varepsilon \delta, k$ is the coefficient of friction. $u_{i}, w_{i}$ are the components of the vectors $\mathbf{u}, \boldsymbol{\Omega}$ at each point of the contact spot in this basis.

It should be noted that if we go over to spherical coordinates

$$
\left\{\begin{array}{c}
\delta u=r \cos \theta_{1} \\
\omega_{I}=r \sin \theta_{1} \cos \theta_{2} \\
\omega_{I I}=r \sin \theta_{1} \sin \theta_{2} \cos \theta_{3} \\
\omega_{I I I}=r \sin \theta_{1} \sin \theta_{2} \sin \theta_{3}
\end{array},\right.
$$

it can be seen that the expressions for the forces and torques do not depend on $r$ (which is a variation of the ideas proposed in [9]). Thus, taking into account the dependence of $\mathbf{f}$ and $\boldsymbol{\mu}$ on the angles $\theta_{\mathrm{k}}$ (mode angles), we can regard them as functions defined on the sphere $\mathrm{S}^{3}$.

\section{Motion planning}

\subsection{Robot's rotation around the vertical}

Firstly, consider the case when the center of mass of the device is stationary, and the sphere rotates around the vertical. Thus, the problem of changing the course angle without a shift of the center of mass is solved. For mode angles, this assumption corresponds to the conditions $\theta_{1}=\theta_{2}=\theta_{3}=\frac{\pi}{2}$. In this case, a pure spinning mode realizes, where all the components of forces and torques, except for $\mu_{z}$, are equal to zero. Friction torque of rotation is $\mu_{z}=\mu_{z}^{0}=$ const $=\mu$. Thus, it is right:

$$
\alpha=\frac{1}{\mathrm{c}}(\mu t E-\omega I) e=\frac{1}{\mathrm{c}} X e .
$$

Here, the $\boldsymbol{e}$-vector of the vertical, written in the moving coordinate system.

Assuming that the flywheel is driven by a standard electric motor and || $\boldsymbol{\alpha}|| \gg\|\boldsymbol{\omega}\|$, we obtain

$$
c \dot{\alpha}=\kappa_{1} U-\kappa_{2} \alpha .
$$

Where $\kappa_{1,2}$-constant parameters of the motor, vector $\boldsymbol{U}$-vector of control voltages. Consequently,

$$
U=\frac{1}{\kappa_{1}}\left(\dot{X}+\frac{\kappa_{2}}{c} X\right) e
$$

Let us give an example of control voltage graphs for rotating the ball at an angle of $\frac{\pi}{2}$ in 4 seconds, choosing motor's parameters $\kappa_{1}=6, \kappa_{2}=0.06$. Robot's parameters are: $M=4, m=0.612, R=0.2, a=$ $0.1, v=54.46$. Vector $\boldsymbol{e}=(1 / \sqrt{3} ; 1 / \sqrt{3} ; 1 / \sqrt{3})$ 
Note, that a constant friction torque $\mu$ can be considered as a moment of static friction. This value depends only on the roughness parameter of the $\delta$ plane. For example, this value for motor Maxon RE-10 is around 0.02 .

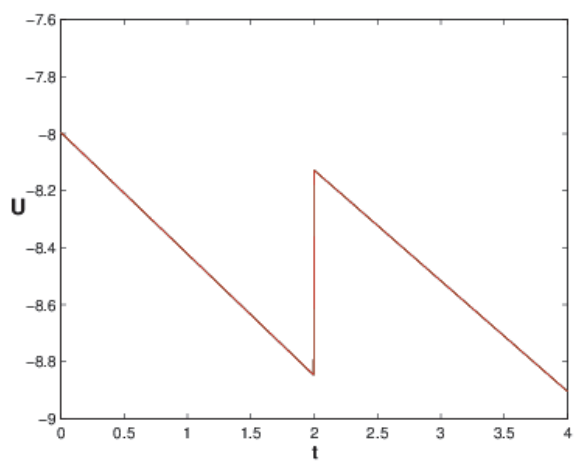

Fig. 1. Control voltage in rotation mode. All voltages are equal to each other.

\subsection{Movement along the line}

One of the most important tasks is to study the dynamics of the robot, when its center of mass moves along a straight line. Without loss of generality, assume that this line is directed to the axis $S x$. Within the framework of the proposed model, slip rolling takes place. This means that the force and the torque are functions of the angle $\theta_{1}$, which we will denote as $\theta$ in this part. The system of equations takes the form ( $\boldsymbol{e}$ is vector $\boldsymbol{e}_{\boldsymbol{y}}$ in the moving coordinate system)

$$
\left\{\begin{array}{c}
\dot{v}=f(\theta) \\
\dot{\omega} I e+c \dot{\alpha}+\omega[e ; \omega I e+c \alpha]=\mu(\theta) e \\
v=r\left(\frac{1}{\delta} \cos \theta+\sin \theta\right)
\end{array}\right.
$$

For forces and torques, as functions $\theta$, the following statements are right (their proof can be found in [10]):

1. $f(\theta) \in C(0 ; \pi)$ and increases on this interval;

2. $\exists \theta_{f} \in\left(\frac{\pi}{2} ; \theta_{*}\right) f\left(\theta_{f}\right)=0, \quad \theta_{*}=\pi-\operatorname{arctg}\left(\frac{1}{\delta}\right)$;

3. $\mu^{0}(\theta) \in C(0 ; \pi)$ And increases on interval $\theta \in\left(\frac{\pi}{2} ; \pi\right)$ and decreases if $\theta \in\left(0 ; \frac{\pi}{2}\right)$;

4. $\mu(\theta) \in C(0 ; \pi)$ and decreases on $\theta \in\left(0 ; \theta_{*}\right)$.

The value $\mu\left(\theta_{f}\right)$ thus can be identified as the moment of static friction. So, the maximum torque on the motor must be greater than this value to allow the motion of the system. This value depends on the contact parameters. An example of areas of possibility of the motion for two real motors (movement possible inside the curve) is below (Fig. 2).

We note that in the framework of this model, the choice of the function $\mathrm{v}$ should be done in the class $C^{1}$, because the discontinuous function with respect to the accelerations leads to discontinuities in velocities. Therefore, to construct control algorithms, we set:

$$
v=A \cos \frac{\eta t}{2} \sin \eta t, \quad t \in\left[0 ; \frac{\pi}{\eta}\right] \text {. }
$$

Below (Fig.3), we give examples of control voltages for the moving of the segment $2 \pi$ during $7 \mathrm{~s}$ if the vector $\mathrm{e}=(1 / \sqrt{3} ; 1 / \sqrt{3} ; 1 / \sqrt{3})$.

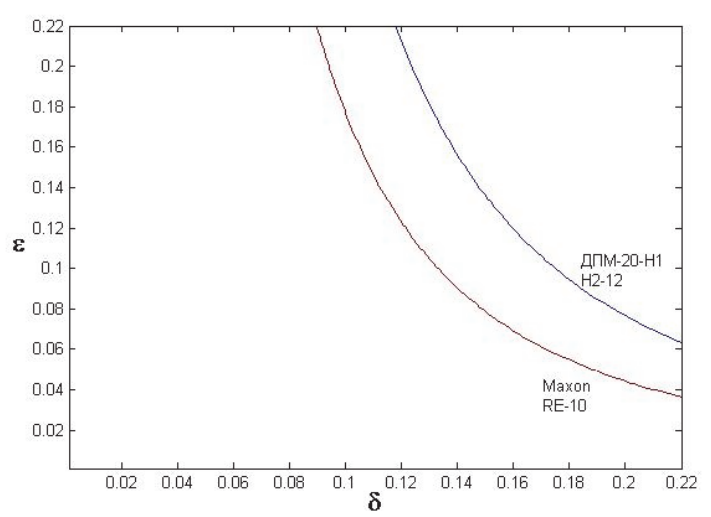

Fig. 2. Area of motion availability.

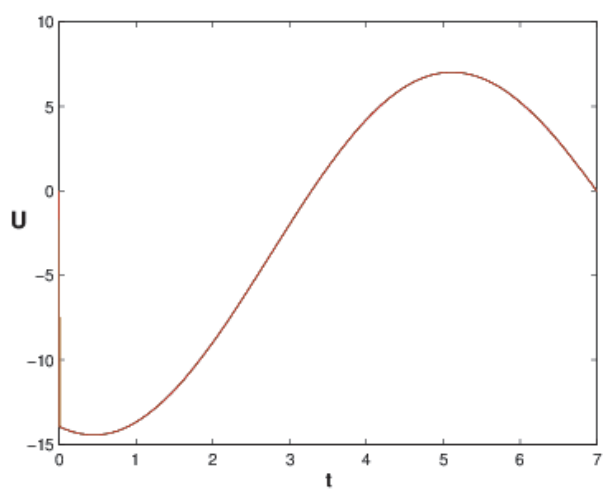

Fig. 3. Control voltage in motion along straight line mode. All voltages are equal to each other.

\subsection{Curvilinear motion}

In spite of the fact that movement on any rectifiable curve can be approximated by movement along broken lines, from the practical point of view such movement is often inefficient. When accuracy of the approximation of the desired trajectory increases, the more the number of segments will have to pass through the robot, and it is necessary to take into account the stop at the end of each segment. Thus, the curvilinear trajectories should be added to the list of alphabetic motions.

First of all, it should be taken into account that for the motion along a curvilinear trajectory, the condition $\Omega_{I}=0$ can be satisfied only on a set of measure zero, since the opposite provides a straight-line trajectory. We associate with the curve along which the center of the sphere moves the natural Frenet basis. Denoting by $\varphi$ the angle between the vectors $e_{I}$ and $e_{\tau}$, we obtain the following system of equations in the projection on the axis of the natural basis:

$$
\left\{\begin{array}{c}
\ddot{s}=f_{I} \cos \varphi-f_{I I} \sin \varphi \\
k(s) \dot{s}^{2}=f_{I} \sin \varphi+f_{I I} \cos \varphi
\end{array}\right.
$$


Here $s$ is a natural parameter, $\mathrm{k}(\mathrm{s})$ is the curvature of the curve. The analysis of forces and torques [10] shows that in the first approximation, as before, we can state $\mathrm{f}$ and $\mu$ determining only by one essential component, for the force $f_{I}$, for the torque $-\mu_{I I}$. Below, the dependence $\mathrm{f}$ and $\mu$ functions of $\theta_{1}, \theta_{2}$ is shown (Fig. 4-8). As a result, we obtain the following system, to find $\varphi, \theta_{1}, \theta_{2}$, after obtaining the required values of $k(s), s(t)$,

$$
\left\{\begin{array}{c}
\ddot{s}=f_{I}\left(\theta_{1}, \theta_{2}\right) \cos \varphi \\
k(s) \dot{s}^{2}=f_{I}\left(\theta_{1}, \theta_{-} 2\right) \cos \varphi \\
\operatorname{tg} \varphi=\frac{-\delta \sin \theta_{1} \cos \theta_{2}}{\delta \sin \theta_{2} \sin \theta_{1}+\cos \theta_{1}}
\end{array}\right.
$$

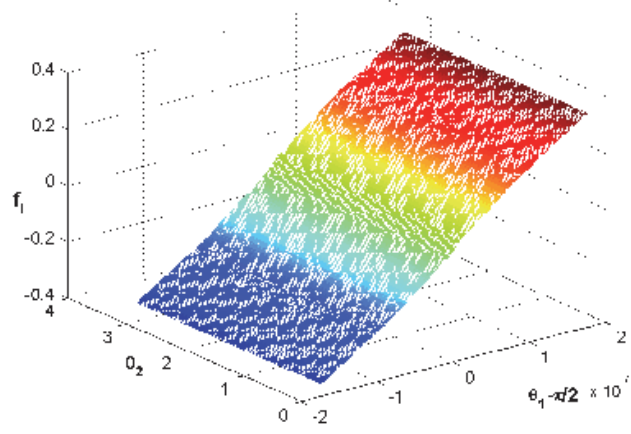

Fig. 4. Surface $f_{I}\left(\theta_{1}, \theta_{2}\right)$.

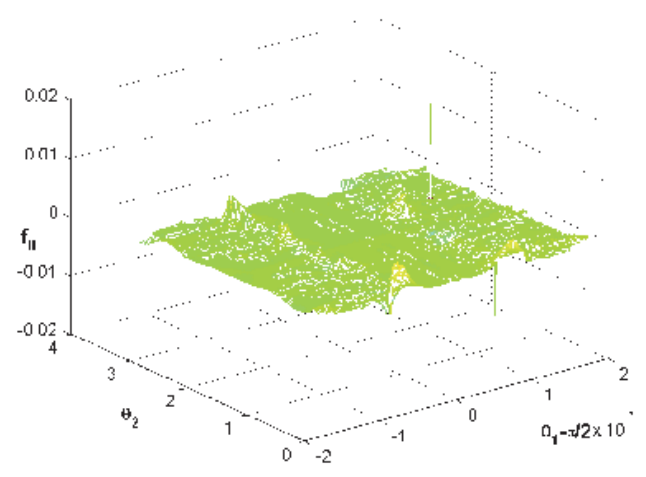

Fig. 5. Surface $f_{I I}\left(\theta_{1}, \theta_{2}\right)$.

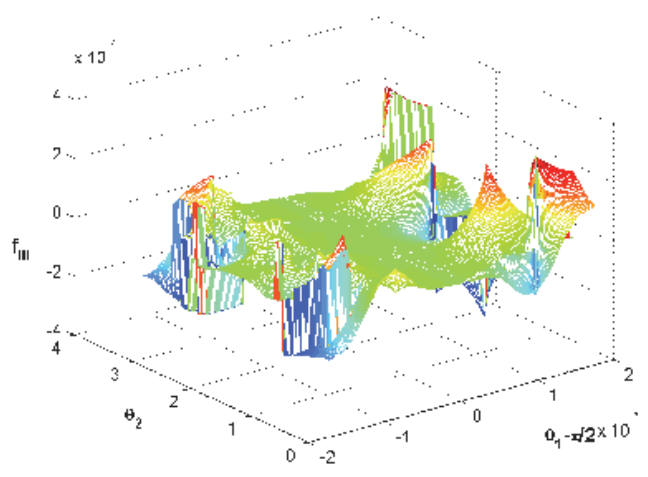

Fig. 6. Surface $f_{I I I}\left(\theta_{1}, \theta_{2}\right)$.

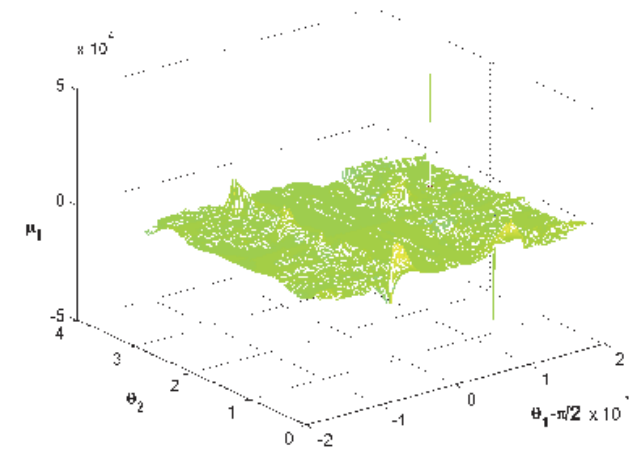

Fig. 7. Surface $\mu_{I}\left(\theta_{1}, \theta_{2}\right)$.

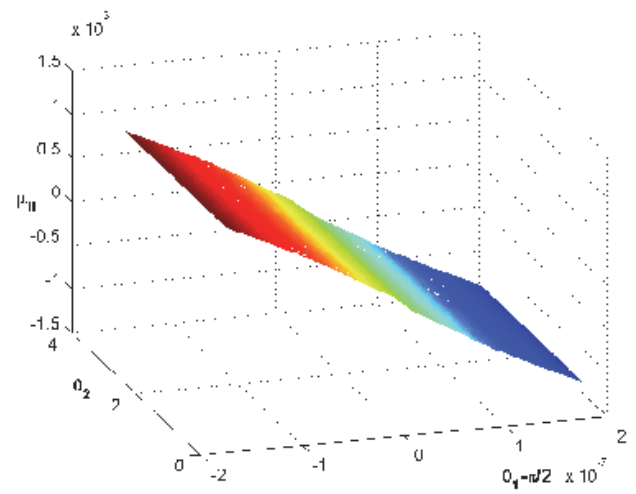

Fig. 8. Surface $\mu_{I I}\left(\theta_{1}, \theta_{2}\right)$.

The $s(t)$ dependence is chosen by the formula:

$$
\dot{s}(t)=A \cos \frac{\eta t}{2} \sin \eta t, \quad t \in\left[0 ; \frac{\pi}{\eta}\right] .
$$

Examples of control voltages when moving along a quarter of a circle of radius $\rho=4$ in 7 seconds (Fig. 9).

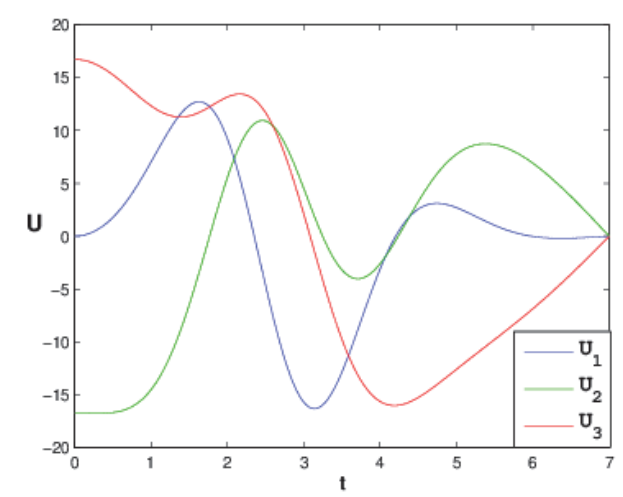

Fig. 9. Control voltages on motion along circle arc.

\section{Conclusion}

The developed mechanical model of the spherical robot solves the task of motion control of a sphere on various trajectories. The movements from rotation on the point and the movements on a straight line piece before the basic movements on curvilinear trajectories are implemented. Experimental results proved technical 
possibility of realization of a sphere robot with flywheels.

\section{References}

1. Electronic resource http://www.rotundus.se/

2. V.E. Pavlovsky, G.P. Terekhov Special equipment and communication, 3, 19-25 (2012)

3. A.V. Borisov, A.A. Kilin, I.S. Mamaev, Nonlinear dynamics, 8(2), 289-307 (2012)

4. A.V. Borisov, A.A. Kilin, I.S. Mamaev, Mobile robots: a robot-wheel and a robot-ball, 131-168, (M.-Izhevsk: Institute for Computer Research, 2013)

5. P. Contensou, Problems of gyroscopy, 60-77 (M.: Mir, 1967).

6. V.F. Zhuravlev, PMM, 62(5), $762-767$ (1998)

7. A.V. Karapetyan, PMM, 73(4), 515-519 (2009)

8. A.V. Karapetyan, PMM, 74(4), 531-535 (2010).

9. T.V. Salnikova, D.V. Treshchev, S.R. Gallyamov. Nonlinear dynamics, 8(1), 83-101 (2012)

10. G.P. Terekhov, V.E Pavlovsky. Preprints of KIAM 16(31) (2017) 